\title{
Empleo y demanda efectiva en la actividad textil, prendas de vestir e industria del cuero de la economía mexicana
}

\author{
Rogelio Varela Llamas* \\ Ramón Castillo Ponce**
}

Resumen. El presente trabajo analiza el impacto de la demanda efectiva sobre el nivel de ocupación en las 51 clases de actividad económica que conforman el subsector 32 dela industria manufacturera en México. Específicamente, se procura identificar las clases de actividad económica que presentan una probabilidad aceptable de potenciar el empleo por encima del promedio del subsector. Para tal efecto, se estiman tres modelos de elección discreta: uno de probabilidad lineal, un Logit y un Probit. Esto con el interés de contrastar resultados alternativos y seleccionar el modelo más idóneo para explicar la relación analítica indicada. Los resultados empíricos indican que el modelo Probit exhibe el mejor ajuste para explicar la capacidad de generar empleos.

Palabras clave: empleo, demanda efectiva, modelo de probabilidad lineal, modelo Logit, modelo Probit.

\begin{abstract}
A bstract. This document analyses the impact of effective demand on employment level of the 51 classes of economic activity, which conform subsector 32 of the manufacturing industry in Mexico. Specifically, the document identifies the classes of economic activity that represent an acceptable probability of generating employment above the subsector's average. To that end, three models of discrete choices are estimated: a linear probability model, a Logit model, and a Probit model. The estimations are performed with the purpose of comparing alternative results and selecting the most efficient model to explain the statistical relationship previously mentioned. The empirical results indicate that the Probit model exhibits the best fit to explain the capacity of generating jobs.

Keywords: employment, effective demand, linear probability model, Logit model, Probit model.
\end{abstract}

\footnotetext{
* Docente dela Facultad de Economía. Universidad Autónoma de Baja California. Correo electrónico: varelall@uabc.mx

** Docente dela Facultad de Economía. Universidad Autónoma de Baja California. Correo electrónico: rcastil@calstatela.edu
} 


\section{Introducción}

El empleo es una variable fundamental que, además de permitir evaluar el grado de estabilidad macroeconómica, también ayuda a valorar los niveles de bienestar social alcanzados por un país, región o sector económico. La teoría del empleo que los distintos paradigmas han desarrollado a lo largo de la historia del pensamiento económico constituyeel marco conceptual y analítico quepermiteabordar el tema de la ocupación laboral como objeto de estudio. En esta perspectiva, el presente trabajo tiene como objeto analizar la relación teórica entre empleo y demanda efectiva en el subsector 32 de la industria manufacturera de M éxico, asociado a la actividad de textiles, prendas de vestir e industria del cuero. El análisis se realiza para 1998, último año del cual se dispone información en el censo industrial. Delos nuevesubsectores que componen la tercera gran división dela economía nacional, el 32 es uno de los que más capacidad ha mostrado para generar empleos, razón por la cual fueel egido como objeto de análisis.

Al medir la sensibilidad del empleo ante los cambios presentados en la demanda efectiva en cada una de las 51 clases deactividad económica queconforman el subsector 32, se procura identificar aquellas actividades que tienen probabilidad de generar empleos a un nivel superior e inferior del promedio del subsector. Esto permitirá efectuar una clasificación de las clases de actividad económica según su potencial para generar empleos en función de su nivel de demanda efectiva.

El trabajo se estructura en cuatro apartados, el primero de los cuales traza una descripción de los planteamientos centrales del paradigma keynesiano en torno a los determinantes del empleo, con el interés de justificar teóricamente la especificación y estimación de la ecuación de regresión. En el segundo apartado se enuncian las fuentes de información y el tratamiento que se le dio a la misma para ser incorporada en los modelos probabilísticos binarios. En el tercero se reportan los resultados de la estimación de un modelo de probabilidad lineal (MPL), así como su interpretación y valoración. Finalmente, en el cuarto apartado se analizan resultados al ternativos, obtenidos a partir 
de la estimación de modelos Logit y Probit, mismos que se contrastan con los obtenidos por el MPL.

\section{M arco de referencia}

La teoría keynesiana es uno de los enfoques teóricos más utilizados para explicar la evolución del empleo. Desde los años cuarenta hasta los setenta del siglo xx, su influencia en el campo de la economía normativa fue decisiva en virtud de que marcó las pautas para la formulación einstrumentación delas políticas públicas antirecesivas (Barber, 1998). A partir del ascenso de las ideas neoclásicas y de su aplicación en las décadas de los ochenta y noventa, en diversos ámbitos de la vida académica e institucional se pensó que la capacidad explicativa del keynesianismo se había agotado dado el acotamiento del tamaño del Estado y la cada vez menos activa política de gasto público.

En estecontexto, en los siguientes párrafos seexplica en forma breveel paradigma keynesiano y en particular el principio dela demanda efectiva a fin de disponer de un marco teórico que le dé sentido al trabajo empírico. Una crítica fundamental que Keynes siempre dirigió en contra dela corrienteneoclásica es queel mercado laboral no se autorregula a partir del comportamiento del salario real, lo cual implica que la economía no siempre está en equilibrio y con pleno empleo (Anisi, 1988). Esto obedecea queexisten rigideces quecomplican el proceso deajusteautomático. Keynes sostenía quelos planteamientos del paradigma neoliberal se contraponen con lo queaconteceen la realidad. En particular, afirmaba que había una distancia entre los hechos y la real idad, es decir, entre el mundo ideal izado por la teoría y el funcionamiento real del mercado laboral. Una de sus principales preocupaciones fue describir el mecanismo que causa el desempleo involuntario y que pone en cuestionamiento a la ortodoxia liberal y a la lógica de que únicamente hay desempleo involuntario cuando el Estado interviene en la economía.

Se plantea entonces que la reducción del empleo se atribuye a una insuficiencia de demanda efectiva derivada de una descoordinación entre los agentes económicos que real izan el gasto de consumo e inversión. Cuando el nivel de demanda efectiva se encuentra por deba- 
jo de lo requerido para generar el pleno empleo, se produce la desocupación involuntaria y, por ende, la inexistencia del equilibrio en el mercado laboral (McConnell y Brue, 2002). En esta perspectiva, se considera que las personas y las unidades familiares efectúan el gasto de consumo y el empresariado el gasto de inversión.

La propuesta de Keynes como respuesta al problema del desempleo consisteen queel Estado, mediante una política fiscal expansiva, puede contribuir a que la economía crezca con el impulso de la demanda efectiva (Fremman y Soete, 1996). Detrás de esteplanteamiento subyacela preocupación deque, al producir déficit presupuestario, el aumento del gasto gubernamental puedeinducir al alza el nivel de precios y complicar el objetivo de un crecimiento sostenido de largo plazo (Ekelum y Herber, 1997). En definitiva se asume queel desempleo involuntario es producto de que las empresas en condiciones de recesión o reducida demanda efectiva no están dispuestas a producir más, debido a que esto aumenta la variación de existencias y reduce el nivel de beneficios. Por ello no es fortuito que en periodos de desaceleración económica, las empresas maximicen las ganancias por medio de la expulsión de fuerza de trabajo (Aláez, 1998).

De acuerdo con Keynes (1990), los factores de rigidez son los que no permiten que los movimientos salarial es ajusten el mercado hasta desaparecer el desempleo involuntario. Se creeque los sindicatos, como factor de rigidez, difícilmente van a acceder a una reducción de los salarios nominales, pues ello se traduciría en una reducción de los salarios reales que impactaría en forma directa y en sentido contrario sobre los logros dela negociación colectiva ya al canzados. Desde esta lógica se infiere una escasa probabilidad de que los salarios reales sean flexibles, ya que la negociación colectiva puede actuar como un mecanismo de rigidez que ubique los salarios nominales por encima de lo que el propio mercado determinaría. En el modelo keynesiano la función de oferta de trabajo no es creciente con el salario real , sino con el salario monetario, pues se supone que los trabajadores están influidos por lailusión monetaria. Seplantea queun agente económico actúa con ilusión monetaria cuando reacciona antel os cambios delas variables nominales, aun cuando no haya tenido lugar ningún cambio real en su situación (Ehrenberg y Smith, 1999). 
En un plano más formal, el empleo en el planteamiento keynesiano seentiendeen el contexto deuna función deproducción y deun equilibrio entreproducción y demanda agregada. Cuando el nivel deproducción de una economía se encuentra por debajo del pleno empleo con una determinada demanda agregada, la única forma de acercarse a éste es incrementando la demanda, de manera que no haya variación de existencias que reflejen una insuficiencia de demanda efectiva. En esta visión, las empresas más que desear una reducción delos salarios, lo que prefieren son suspensiones temporales en aras de minimizar sus costos.

El análisis del empleo gira alrededor del concepto de desempleo involuntario. Seconcibeel pleno empleo como la ausencia dedesempleo involuntario; sin embargo, al igual que en el modelo neoclásico también se reconoce que puede haber desempleo voluntario, friccional o presumiblemente cíclico o estructural. El desempleo involuntario se manifiesta debido a que una parte importante de la población que está en edad de trabajar y que, por supuesto, desea incorporarse al mercado, no puede hacerlo porque no existe la suficiente demanda efectiva, entendida como la diferencia entrela producción bruta total y la variación de existencias.

Cuando el origen del desempleo es una demanda efectiva insuficiente, se suele hablar de desempleo keynesiano involuntario y la política idónea para resolverlo es aquella que se basa en el incremento del gasto público. Esto implica la existencia de estímulos al aumento de la producción, de manera quese entreen un círculo virtuoso en el que los aumentos de la demanda efectiva conduzcan a un aumento del crecimiento económico y, en consecuencia, del empleo. Sólo una recuperación de la producción permitiría que los desempleados fueran contratados. A pesar de los efectos positivos que produce un aumento de la demanda, también pueden generarse efectos adversos como la existencia de mayores tensiones en el mercado de trabajo, lo que propiciaría un aumento en los sal arios que, a su vez, podría generar aumentos en los costos de producción de las empresas y sobre los precios de los bienes y servicios, desatándose con ello una espiral inflacionaria. 


\section{Fuentes de información}

La información estadística que seutilizó en las estimaciones aquí presentadas tiene como fuente primaria al Instituto Nacional de Estadística, Geografía e Informática (INEGI) y es presentada en el cuadro 1. Los datos provienen del censo industrial que dicho instituto realizó en 1998 y que fueron publicados en el año 2000. Las variables expuestas en dicho cuadro corresponden al subsector 32 de la industria manufacturera.

La variable de personal ocupado, de acuerdo al propio censo, incluye a hombres y mujeres que dependen de la unidad económica y trabajan bajo su dirección y control, y que cubren como mínimo una tercera parte de la jornada laboral recibiendo regularmente un pago. ${ }^{1}$ En general, se incluye a los obreros vinculados con el proceso de producción y toda su logística, y la fuerza de trabajo que reúne mayores niveles de calificación y quese relaciona con las tareas de administración y gestión empresarial. A mbos conceptos están expresados en miles de ocupados para cada una de las 51 clases de actividad económica. Para calcular la demanda efectiva se consideró la producción bruta total contabilizada a precios nominales y la variación de existencias, definida estaúltima como la producción no absorbida por el mercado que, al ser almacenada, produce costos de operación y mantenimiento, o bien es el valor queresulta derestar al inventario final el inventario inicial. La demanda efectiva se aproximó a través de la diferencia entre producción bruta total y variación de existencias, deflactadas ambas variables con baseen el índicenacional depreciosal productor de 1994.

Aunque no hay una diferencia significativa entre dicho índicey el de precios al consumidor para el año en cuestión, se optó por utilizar el índicedepreciosal productor porqueestáinvolucrada en el cálculo

\footnotetext{
${ }^{1}$ Incluye personal de la unidad económica que trabajó fuera de la misma bajo su control laboral y legal, trabajadores en huelga, personal con licencia, vacaciones y con licencia temporal. Excluye a los pensionados y personal que prestó servicios a través de honorarios o comisiones.
} 
de la demanda efectiva una variable representativa del esfuerzo de producción queesel producto bruto total. También es importantedestacar que la información presentada en el cuadro 1 se ajusta a una estructura de datos de corte transversal o de sección cruzada, que es justamente lo requerido para estimar model os probabilísticos en donde la variable dependiente es binaria. Esto supone que se tienen $\mathrm{N}$ unidades deanálisis (clases deactividad económica) y T años. En este caso $\mathrm{N}=51$ y $\mathrm{T}=1$, variables que permiten una muestra suficiente para efectuar un análisis de regresión de sección cruzada.

\section{Estimación y análisis de resultados del modelo M PL}

Un primer esfuerzo para realizar un análisis mi croeconométrico entre el empleo y la demanda consisteen estimar un MPL. En éstela variable dependiente $\left(Y_{i}\right)$ serefiereal nivel deempleo y es binaria. Para el conjunto delas 51 clases deactividad económica queseagrupan en dicho subsector, $Y_{i}$ asume valores de (1) cuando la actividad en cuestión genera empleos por encima del promedio del subsector, y valores de (0) cuando son inferiores al mismo. Con el propósito de contrastar la hipótesis que establece que los cambios en la demanda efectiva explican positivamentelas variaciones en el empleo, seformal iza una ecuación de regresión simple para conocer el valor esperado de $Y_{i}$ dada la observación de la variable independiente $X_{i}$, que se refiere a la demanda efectiva real.

Dado un nivel de $X_{i}$, la probabilidad deque una clase de actividad genere empleos por encima del promedio del subsector 32 se representa como:

$$
\operatorname{Pr}=(Y i=1 / X i)=P i \text {. }
$$

En contraste, la probabilidad de quese generen empleos por debajo del promedio referido se denota por:

$$
\operatorname{Pr}=\left(Y i=0 / X_{i}\right)=1-P i
$$


Cuadro 1. Estadísticas del subsector 32 dela industria manufacturera M éxico, 1998.

\begin{tabular}{|c|c|c|c|c|c|c|c|}
\hline \multirow[t]{2}{*}{$\begin{array}{l}\text { Subsector 32: Textiles, prendas } \\
\text { de vestir e industria del cuero }\end{array}$} & \multirow[t]{2}{*}{ Dummy } & \multirow[t]{2}{*}{$\begin{array}{l}\text { Personal } \\
\text { ocupado }\end{array}$} & \multirow[t]{2}{*}{$\begin{array}{l}\text { Producción } \\
\text { total }\end{array}$} & $\begin{array}{l}\text { Variación } \\
\text { de existencias }\end{array}$ & $\begin{array}{l}\text { Demanda } \\
\text { efectiva }\end{array}$ & \multirow{2}{*}{$\begin{array}{l}\text { Demanda } \\
\text { efectiva } \\
1994=100\end{array}$} & \multirow{2}{*}{$\begin{array}{l}\text { LDER } \\
\text { Logaritmo natural } \\
\text { dedemanda } \\
\text { efectiva real }\end{array}$} \\
\hline & & & & \multicolumn{2}{|c|}{ Miles de pesos } & & \\
\hline Clase de actividad económica & & (a) & (b) & (c) & $(d)=(b)-(c)$ & $(e)=(d) / 250,84^{*}$ & \\
\hline 321111. Preparación de fibras de henequén & 0 & 584 & 62378 & 475 & 61903 & 24678.28 & 10.11 \\
\hline $\begin{array}{l}\text { 321112. Hilado y tejido de henequén } \\
\text { 321113. Hilado y tejido de ixtle de palma }\end{array}$ & 0 & 1713 & 230188 & 17333 & 212855 & 84856.88 & 11.35 \\
\hline $\begin{array}{l}\text { y otras fibras duras } \\
321120 \text {. Fabricación de cordelería de fibras }\end{array}$ & 0 & 10135 & 187558 & 5329 & 182229 & 72647.50 & 11.19 \\
\hline de todo tipo naturales o químicas & 0 & 4280 & 583235 & 16044 & 567191 & 226116.6 & 12.33 \\
\hline 321201. Despepite y empaque de algodón & 0 & 2028 & 746336 & 20192 & 726144 & 289484.9 & 12.58 \\
\hline 321202. Hilado de fibras blandas & 0 & 13640 & 3953153 & 55209 & 3897944 & 1553956 & 14.26 \\
\hline $\begin{array}{l}\text { 321203. Fabricación de hilo para coser, bordar y tejer } \\
\text { 321204. Fabricación de estambres de lana }\end{array}$ & 0 & 10881 & 4734548 & 128679 & 4605869 & 1836178 & 14.42 \\
\hline 321205. Fabricación de telas de lana y sus mezclas & 0 & 3445 & 1442368 & 71331 & 1371037 & 546578.3 & 13.21 \\
\hline 321206. Tejido de fibras blandas & 1 & 44791 & 16482370 & 382052 & 16100318 & 6418561 & 15.67 \\
\hline $\begin{array}{l}\text { 321207. A cabados de hilos y telas de fibras blandas } \\
\text { 321208. Fabricación de encajes, cintas, etiquetas }\end{array}$ & 1 & 20099 & 3373772 & 76969 & 3296803 & 1314305 & 14.09 \\
\hline $\begin{array}{l}\text { y otros productos de pasamanería } \\
321209 \text {. Fabricación de fieltro y entretelas }\end{array}$ & 0 & 8931 & 1739187 & 33468 & 1705719 & 680002.8 & 13.43 \\
\hline defibras blandas & 0 & 1482 & 582467 & 915 & 581552 & 231841.8 & 12.35 \\
\hline 321210. Tejido de rafia sintética & 0 & 5717 & 909116 & 38692 & 870424 & 347003.7 & 12.76 \\
\hline
\end{tabular}


321211. Tejido de redes y paño para pescar de fibras blandas

321212. Hilado y tejido de regenerados

321214. Fabricación de al godón absorbente,

vendas y similares

321215. Fabricación de telas no tejidas

321216. Fabricación de textiles recubiertos

o con baño

321311. Confecciones de sábanas, manteles,

colchas y similares

321312. Confección de productos bordados

y deshilados

321321. Confección de toldos, cubiertas

para automóvil y tiendas de campaña

321322. Confección de otros artículos con

materiales textiles naturales o sintéticos

321331. Tejido a mano de alfombras y tapetes

de fibras blandas

321332. Tejido a máquina de alfombras y tapetes

de fibras blandas

321401. Fabricación de medias y calcetines

321402. Fabricación de suéteres

321403. Fabricación de ropa interior

de punto

321404. Fabricación de telas de punto

321405. Fabricación de ropa exterior

de punto y otros artículos

\begin{tabular}{rrrrrrr}
0 & 6263 & 223313 & 5831 & 217482 & 86701.48 & 11.37 \\
0 & 4929 & 803059 & 23211 & 779848 & 310894.6 & 12.65 \\
0 & 4200 & 815118 & 5084 & 810034 & 322928.6 & 12.69 \\
0 & 2751 & 1554213 & 66945 & 1487268 & 592915 & 13.29 \\
0 & 2711 & 1082204 & 29947 & 1052257 & 419493.3 & 12.95 \\
0 & 17499 & 4222435 & 151773 & 4070662 & 1622812 & 14.30 \\
0 & 6309 & 438242 & 15704 & 422538 & 168449.2 & 12.03 \\
0 & 3744 & 641234 & 9954 & 631280 & 251666.4 & 12.44 \\
1 & 51568 & 13910023 & 134065 & 13775958 & 5491930 & 15.52 \\
0 & 1776 & 67251 & 1300 & 65951 & 26292.06 & 10.18 \\
0 & 2567 & 1800525 & 70168 & 1730357 & 689825 & 13.44 \\
1 & 18208 & 3281548 & 17102 & 3264446 & 1301406 & 14.08 \\
0 & 6791 & 816087 & 24042 & 792045 & 315757.1 & 12.66 \\
0 & 2758 & 472077 & 15178 & 456899 & 182147.6 & 12.11 \\
0 & 7089 & 3377101 & 164889 & 3212212 & 1280582 & 14.06 \\
1 & 18136 & 2010374 & 60315 & 1950059 & 777411.5 & 13.56 \\
\hline
\end{tabular}

Continúa... 
Cuadro 1. Estadísticas del subsector 32 de la industria manufacturera M éxico, 1998.

\begin{tabular}{|c|c|c|c|c|c|c|c|}
\hline \multirow[t]{2}{*}{$\begin{array}{l}\text { Subsector 32: Textiles, prendas } \\
\text { de vestir e industria del cuero }\end{array}$} & \multirow[t]{2}{*}{ Dummy } & \multirow[t]{2}{*}{$\begin{array}{l}\text { Personal } \\
\text { ocupado }\end{array}$} & \multirow[t]{2}{*}{$\begin{array}{l}\text { Producción } \\
\text { total }\end{array}$} & $\begin{array}{c}\text { Variación } \\
\text { de existencias }\end{array}$ & $\begin{array}{l}\text { Demanda } \\
\text { efectiva }\end{array}$ & \multirow{2}{*}{$\begin{array}{l}\text { Demanda } \\
\text { efectiva } \\
\text { 1994=100 }\end{array}$} & \multirow{2}{*}{$\begin{array}{l}\text { LDER } \\
\text { Logaritmo natu } \\
\text { dedemanda } \\
\text { efectiva real }\end{array}$} \\
\hline & & & & \multicolumn{2}{|c|}{ Miles de pesos } & & \\
\hline Clase de actividad económica & & (a) & (b) & (c) & $(d)=(b)-(c)$ & $(e)=(d) / 250,84^{*}$ & \\
\hline $\begin{array}{l}\text { 322001. Confección de ropa exterior } \\
\text { para caballero hecha en serie } \\
\text { 322002. Confección de ropa exterior }\end{array}$ & 1 & 163061 & 15953889 & 461330 & 15492559 & 6176271 & 15.64 \\
\hline $\begin{array}{l}\text { para caballero hecha sobre medida } \\
322003 \text {. Confección de ropa exterior }\end{array}$ & 0 & 13055 & 722634 & 10142 & 712492 & 284042.4 & 12.56 \\
\hline $\begin{array}{l}\text { para dama hecha en serie } \\
322004 \text {. Confección de ropa exterior }\end{array}$ & 1 & 95854 & 10025119 & 601026 & 9424093 & 3757014 & 15.14 \\
\hline para dama hecha sobre medida & 0 & 14213 & 774803 & 28193 & 746610 & 297643.9 & 12.60 \\
\hline 322005. Confección de camisas & 1 & 30918 & 2531286 & 32950 & 2498336 & 995987.9 & 13.81 \\
\hline $\begin{array}{l}\text { 322007. Confección de prendas de vestir de cuero, } \\
\text { piel y materiales sucedáneos para hombre } \\
\text { 322008. Confección de prendas de vestir }\end{array}$ & 0 & 1438 & 273784 & 8493 & 265291 & 105761 & 11.57 \\
\hline $\begin{array}{l}\text { de cuero, piel y materiales sucedáneos para hombre } \\
322009 \text {. Confección de ropa exterior }\end{array}$ & 0 & 138 & 12486 & 218 & 12268 & 4890.767 & 8.50 \\
\hline para niños y niñas & 1 & 25643 & 2854499 & 115898 & 2738601 & 1091772 & 13.90 \\
\hline
\end{tabular}


322010. Confección de otras prendas

exteriores de vestir

322011. Confección de corsetería

322012. Confección de otra ropa interior

322013. Fabricación de sombreros,

gorras y similares

322014. Fabricación de somb., gorras y similares

hechos de palma y otras fibras duras

322015. Confección de guantes, corbatas,

pañuelos y similares

323001. Curtido y acabado de cuero

323002. Curtido y acabado de pieles sin depilar

323003. Fabricación de productos de cuero,

piel y materiales sucedáneos

324001. Fabricación de calzado

principalmente de cuero

324002. Fabricación de calzado de tela

con suela de hule o sintética

324003. Fabricación de huaraches, al pargatas

y otro tipo decalzado no espec. anteriormente

$\begin{array}{rrrrrrr}0 & 2532 & 88049 & 6311 & 81738 & 32585.71 & 10.39 \\ 1 & 39224 & 3965806 & 89756 & 3876050 & 1545228 & 14.25 \\ 0 & 16362 & 2005285 & 57262 & 1948023 & 776599.8 & 13.56 \\ 0 & 2395 & 187885 & 7362 & 180523 & 71967.39 & 11.18 \\ 0 & 2701 & 138548 & 7225 & 131323 & 52353.29 & 10.87 \\ 0 & 2708 & 300464 & 17593 & 282871 & 112769.5 & 11.63 \\ 0 & 9930 & 4254011 & 83749 & 4170262 & 1662519 & 14.32 \\ 0 & 4335 & 1188015 & 61326 & 1126689 & 449166.4 & 13.02 \\ 1 & 28652 & 3324478 & 101833 & 3222645 & 1284741 & 14.07 \\ 1 & 87439 & 12050468 & 228870 & 11821598 & 4712804 & 15.37 \\ 0 & 13842 & 1677493 & 41678 & 1635815 & 652134.8 & 13.39 \\ 0 & 6821 & 526936 & 9093 & 517843 & 206443.5 & 12.24\end{array}$

DEMANDA EFECTIVA PROMEDIO (logaritmos)

12925

Fuente: Cálculos propios con base en el Censo Industrial 1998, INEGI.

*índice $\mathrm{N}$ acional de Precios al Productor, promedio anual. 
La definición de la esperanza matemática puede ilustrarse como:

$$
E\left(Y_{i} / X_{i}\right)=O\left(1-P_{i}\right)+1\left(P_{i}\right)=P_{i}
$$

misma quees análoga a la ecuación $E\left(Y_{i} / X_{i}\right)=\beta_{1}+\beta_{2} X_{i}=$ Pi debido a que $0(1-\mathrm{Pi})=0$. Si la probabilidad de que el evento ocurra $(Y \mathrm{i}=1$ / $X 1)$ debe estar entre 0 y 1 , se deduce quela variable dependiente estará sujeta a la restricción $0 \leq E\left(Y_{i} / X_{i}\right) \leq 1$ (Gujarati, 2004).

A priori, se esperaría que las estimaciones de las probabilidades se ubicaran en el rango [0, 1]. Sin embargo, una limitación en el uso de MPL es que no siempresecumpletal restricción. Una forma desuperar este problema consiste en el iminar las observaciones que caen fuera dedicho intervalo, con la consecuencia dequese reduce el tamaño de la muestra y los grados de libertad. Otra opción consiste en sustituir los val ores estimados negativos de la variable dependiente, $\hat{Y}_{i}$, por 0.01 y los valores mayores que la unidad por 0.99 .

Inicialmente, seestima una regresión por medio dela metodología de Mínimos Cuadrados Ordinarios (MCO). Los resultados muestran, no sorprendentemente, quealgunos val ores estimados se encuentran fuera del rango deseado. En particular, notamos que hay tres valores superiores a la unidad, los cual es corresponden a las clases económicas 321206 (tejido de fibras blandas), 321322 (confección de otros artículos con materiales textiles naturales o sintéticos) y 322001 (confección de ropa exterior para caballero hecha en serie). De acuerdo con lo señalado en el párrafo anterior, se opta por sustituir los valores superiores a la unidad por 0.99. En la figura 1 pueden observarse los valores estimados sin ajustar los tres casos mencionados, mientras que en la gráfica 2 se muestran los valores ya ajustados.

Más allá del problema de rango que se presenta en la estimación del MPL con Mco, es común encontrar en estudios de datos de sección cruzada problemas de heteroscedasticidad. De tal suerte que se opta por transformar la información para estimar los coeficientes mediante la metodología de Mínimos Cuadrados General izados (MCG). Brevemente, asumiendo que la varianza (w) del error estocástico es igual a 
$P_{i}\left(1-P_{i}\right)$ y queE $\left(Y_{i} / X_{i}\right)=\beta_{1}+\beta_{2} X_{i}=P_{i}$, entonces un primer paso para aplicar MCG es obtener:

$\sqrt{w}$, equivalentea $\sqrt{P_{i}\left(1-P_{i}\right)}=\sqrt{E\left(Y_{i} / X_{i}\right)\left[1-E\left(Y_{i} / X_{i}\right)\right]}$

Al dividir cada parte de la ecuación de regresión entre $\sqrt{w}$, se obtienela siguiente expresión:

$Y_{i} / \sqrt{w}=\hat{\beta}_{1}{ }^{*} 1 / \sqrt{w}+\hat{\beta}_{2}{ }^{*} X_{i} / \sqrt{w}+\ldots \ldots+\hat{\beta}_{n}{ }^{*} X_{n} / \sqrt{w}+u_{i}$

Figura 1. Diagrama entre $\hat{Y}_{i}$ y $X_{i}$

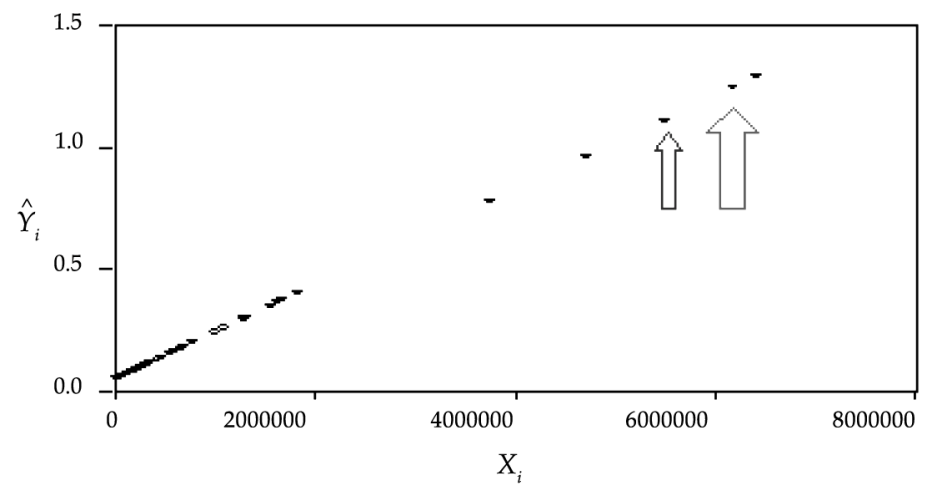

Figura 2. Diagrama ajustado entre $\hat{Y}_{i} \mathrm{y} \mathrm{X}_{\mathrm{i}}$

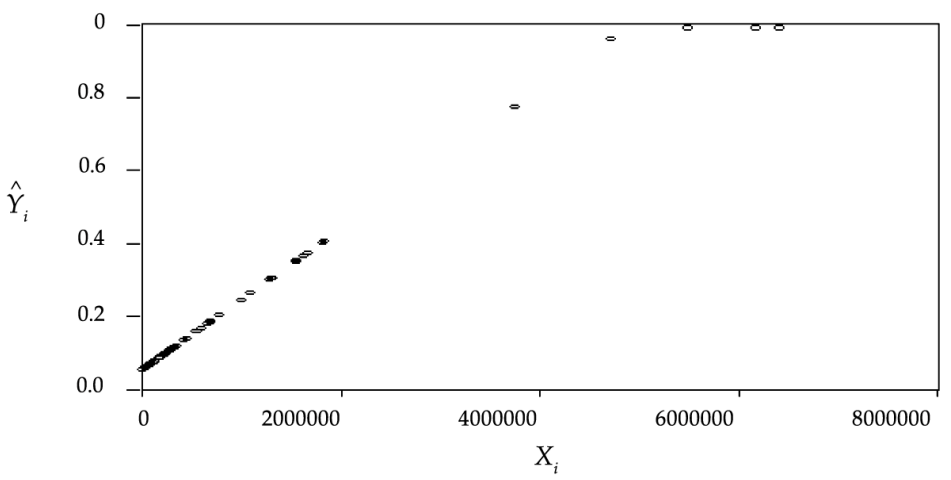


Partiendo de que las estimaciones por mco no indicaron la presencia de varianza homoscedastica, se procedió a estimar el modelo para el nivel de empleo y la demanda real efectiva por medio de la especificación siguiente:

$$
Y_{i} / \sqrt{w}=\hat{\beta}_{1}{ }^{*} 1 / \sqrt{w}+\hat{\beta}_{2}{ }^{*} X_{i} / \sqrt{w}
$$

Para detectar si existe heteroscedasticidad, se aplicó el contraste Arch LM basado en la ecuación de regresión auxiliar $\mathrm{e}^{2}=\rho_{1} *\left(\mathrm{e}_{\mathrm{t}-1}\right)^{2}+$ $\rho_{2}{ }^{*}\left(e_{t-2}\right)^{2}+\ldots \ldots \ldots+\rho_{n} *\left(e_{t-n}\right)^{n}$. Deacuerdo al estadístico $\mathrm{F}$ y su probabilidad mayor a .05 presentados en el cuadro dos, se acepta la hipótesis nula que establece que el modelo presenta varianza constante en los residuos.

Cuadro 2. Prueba dediagnóstico.

\begin{tabular}{lll}
\hline & $\begin{array}{l}\text { Heteroscedasticidad } \\
\text { ContrasteArch LM (-1) }\end{array}$ & $\begin{array}{l}\text { Heteroscedasticidad } \\
\text { Contraste Arch LM (-2) }\end{array}$ \\
\hline F-estadístico & 0.787255 & 0.783806 \\
Probabilidad & 0.782056 & 0.772510 \\
\hline
\end{tabular}

No obstante el contraste Arch LM indica que no está presente el problema de heterocedasticidad y que se tiene un $\mathrm{R}^{2}$ ajustado de 0.90 , un Durbin-Watson de 2.10 y el signo correcto en $\beta_{2}$ con un valor numérico de $165^{08}$, los resultados son dudosos debido a quelas clases de actividad económica 321206, 322001, 321322, 324001 y 322003 presentan probabilidades superiores a la unidad, violando el rango de $0 \leq$ $E\left(Y_{i} / X_{i}\right) \leq 1$ que se establece en los modelos de elección discreta.

\section{Estimación y análisis de resultados de modelos Logit y Probit}

Con el propósito de obtener resultados alternativos mediante la estimación de modelos Logit y Probit con base al método econométrico 
de máxima verosimilitud, se corrió para el primer caso una regresión basada en una función de crecimiento logística

$$
P_{i}=E\left(Y_{i}=1 / X_{i}\right)=\frac{1}{1+\lambda^{-\left(\beta_{1}+\beta_{2}{ }^{*} X_{i}\right)}}=\frac{1}{1+\lambda^{-Z_{i}}}
$$

Donde $\lambda$ representa la base del logaritmo natural y $Z_{i}$ es una función de distribución logística. $Z_{i}$ es un valor numérico que seencuentra entre $(-\alpha y+\alpha)$ y $P_{\mathrm{i}}$ entre0 1 . Detal manera quePi estárelacionada en forma lineal con $\mathrm{Z} i$ y por tanto con $\mathrm{X}$ i. La probabilidad de que un evento ocurra está dada por $\mathrm{Pi}=1 / 1+\lambda^{-z i}$, y de que no ocurra por 1 $P_{i}$. Siguiendo a Pindyck y Rubinfeld (1998), $\left(1+\lambda^{-2 i}\right) P_{i}=1$, y dividiendo entre $\mathrm{P}_{i}$, y restando 1 seobtiene $\lambda^{-z i}=1 / P$ - 1 equivalentea $1-P_{i} / P_{i}$.

Considerando que $\lambda^{-z i}=1 / \lambda^{z i}$, entonces $\lambda^{z i}=P_{i} / 1-P_{i}$, de manera que tomando logaritmo natural en ambos lados se obtiene $\ln \mathrm{P}_{\mathrm{i}} / 1-\mathrm{P}_{\mathrm{i}}$ $=Z_{i}=\alpha+\beta X_{i}$. De esta forma se establece que $L_{i}=\ln P_{i} / 1-P_{i}$ es la razón de las dos probabilidades y es una expresión lineal en $X_{i}$ y en los parámetros. Como se puede observar, en el modelo Logit la variable por explicar en el ecuación de regresión es el logaritmo dela razón de probabilidad, mientras que la función de probabilidad quesubyace en el modelo Logit es la distribución logística (Gujarati, 2004).

A dicionalmente, se estima un modelo Probit a partir de una ecuación de distribución dela normal

$$
Y_{i}=\int_{-\alpha}^{z_{i}} \frac{1}{2 \pi^{1 / 2}} \lambda^{\frac{-s^{2}}{2}} \partial s+\mu_{i}
$$

En forma simplificada, el model o puedeexpresarse como $Y_{i}=\phi(X$, $\beta)+\mu=\phi\left(Z_{i}\right)+\mu_{i}$, con probabilidades $\operatorname{de} \operatorname{Pr}\left(Y_{i}=1 / X_{i}\right)=P_{i}$ y $\operatorname{Pr}\left(Y_{i}=0 /\right.$ $\left.X_{i}\right)=1=P_{i}$, siendo la suma de ambas igual a la unidad. La estimación del modelo cuantifica la probabilidad de elegir la opción $E\left(Y_{i} / X_{i}\right)=$ $\phi\left(X_{i} \beta\right)=P_{i}$. Es importanteanotar quelos coeficientes estimados en los modelos Logit y Probit no reflejan un cambio en la probabilidad de que la variable dependiente asuma el val or de 1, dado un incremento unitario en el regresor $X_{i}$. Sólo representan el sentido de la relación entre la variable dependientey las variables explicativas, pero en términos ordinales y no cardinales. 
Un valor concreto de $\hat{Y}_{i}=\hat{P}_{i}=\phi(X, \hat{\beta})$ mide la probabilidad de que una clase de actividad económica genere empleos superiores al promedio del subsector dado su nivel de demanda efectiva. La derivada parcial

$$
\frac{\partial \phi\left(X_{i} \beta\right)}{\partial X_{k i}}=\phi\left(X_{i} \beta\right) \beta_{k}
$$

donde $\phi\left(X_{i} \beta\right)$ es la función de densidad de la normal (derivada de la función de distribución) igual $\phi\left(Z_{i}\right)$, muestra queel cambio de probabilidad ante variaciones de $X_{k i}$ depende de los valores que tome la función dedensidad en el punto i-ésimo y del estimador del parámetro $\beta_{k}$. Como en estecaso sól o seconsidera el regresor $X_{i}$, si se desea conocer su efecto marginal en el punto medio de las características de las clases de actividad económica se debe cal cular

$$
\frac{\partial \phi\left(X_{i} \beta\right)}{\partial X_{2 i}}=\phi\left(X_{i} \beta\right) \beta_{2}
$$

A continuación se presentan los resultados de estimación de los dos model os. En ambos casos se optó por plantear una ecuación simple donde el regresor es el logaritmo natural de la demanda efectiva real, LN X i, y la variable dependiente es $Y_{i}$. Los resultados obtenidos fueron los siguientes:

M odelo Logit

$$
\begin{aligned}
& Y_{i}=\hat{\beta}_{1}+\hat{\beta}_{2} * L N X_{i} \\
& Y_{i}=-37.48942+2.6651 * L N X_{i} \\
& \text { err est (12.47194) (0.8948) } \\
& \begin{array}{lll}
z & -3.0059 & 2.9784
\end{array}
\end{aligned}
$$

McFadden $\mathrm{R}^{2}=0.553157$, LR statistic (razón deverosimilitud) $=32.0283$ Criterio deSchwarz $=0.6615$, Criterio de Hannan-Quinn $=0.6147$ 


$$
\begin{aligned}
& \text { M odel o Probit } \\
& Y_{i}=\hat{\beta}_{1}+\hat{\beta}_{2} * L N X_{i} \\
& Y_{i}=-22.1345+1.5730 * L N X_{i} \\
& \text { err est (7.0978) (0.5095) } \\
& \begin{array}{lll}
z & -3.1185 & 3.0874
\end{array}
\end{aligned}
$$

McFadden $\mathrm{R}^{2}=0.5633$, LR statistic (razón de verosimilitud) $=32.6166$ Criterio deSchwarz $=0.6499$, Criterio deH annan-Quinn $=0.6031$, Jarque

- Bera $(\mathrm{F}=5.28, \mathrm{Pr}=0.07)$

Para analizar la significancia estadística de ambos modelos, además de considerar el estadístico $z$, que a propósito es aceptable en ambos casos, se utilizó también el estadístico LR de razón de verosimilitud para probar la Ho: $\beta_{2}=0$. Esta prueba se efectuó a partir dela desigualdad probabilística $\operatorname{Pr}=\left(\operatorname{LR} \pi \mathrm{X}_{\alpha}^{2}\right)=1-\alpha$. El valor crítico de $\mathrm{X}_{\alpha}^{2}$ con $\alpha=0.05$ y un grado de libertad (número de regresores) fue de 3.84 .

Como en ambos casos el estadístico LR es superior al valor crítico dela chi-cuadrada, serechazó la Ho deno significancia del parámetro, validándose de esta forma la adecuada especificación de ambos modelos para estimar $\left(P_{i}=Y_{i}=1 / X_{i}\right)$. Como la función de verosimilitud está directamente vinculada con el tamaño de la muestra, y dado que en este caso es relativamente grande, se utilizaron los criterios de Schwarz y deH annan-Quinn para sel eccionar el mejor modelo probabilístico. El criterio de elección consisteen elegir aquel modelo quetenga val ores numéricos más pequeños en ambos estadísticos. Dado que el model o Probit presenta criterios menores relativos a los hal lados en el modelo Logit, seseleccionó como el más idóneo.

En el cuadro 3 se presentan las probabilidades de que una clase de actividad económica determinada genereempleos por arriba del promedio del subsector en general. En dicho cuadro se listan las nueve clases más importantes de las 13 denotadas con $Y_{i}$ que tienen una probabilidad mayor a 0.5 degenerar empleo superiores al promedio, y las cuatro que presentan un probabilidad inferior a la de referencia. A simismo, se indican las cuatro clases de actividad de las 38 denotadas por $Y_{i}=0$ que tienen un probabilidad superior a 0.5 de generar 


\section{Cuadro 3. Probabilidades estimadas mediante el modelo Probit.}

\section{Clase}

Actividades económicas clasificadas con $Y_{i}=1$

(13 clases)

\begin{tabular}{|c|c|c|}
\hline & 9 clases & $\operatorname{Pr}\left(\hat{Y_{i}}=1\right)>0.5$ \\
\hline 321206 & Tejido de fibras blandas & 0.9942 \\
\hline 322002 & Confección de ropa exterior para caballero hecha en serie & 0.9931 \\
\hline 321322 & $\begin{array}{l}\text { Confección de otros artículos con materiales textiles naturales } \\
\text { o sintéticas }\end{array}$ & 0.9886 \\
\hline 32400 & Fabricación de calzado principalmente de cuero & 0.9791 \\
\hline 322003 & Confección de ropa exterior para dama hecha en serie & 0.9535 \\
\hline 322006 & Confección de uniformes & 0.7028 \\
\hline 322011 & Confección de corsetería & 0.6110 \\
\hline 321207 & A cabados de hilos y telas de fibras blandas & 0.5109 \\
\hline \multirow[t]{2}{*}{321401} & Fabricación de medias y calcetines & 0.5047 \\
\hline & 4 clases & $\operatorname{Pr}\left(\hat{Y_{i}}=1\right)<=0.5$ \\
\hline 323003 & $\begin{array}{l}\text { Fabricación de productos de cuero, piel y materiales } \\
\text { sucedáneos }\end{array}$ & 0.4966 \\
\hline 322009 & Confección de ropa exterior para niños y niñas & 0.3957 \\
\hline 322005 & Confección de camisas & 0.3413 \\
\hline 321405 & Fabricación de ropa exterior de punto y otros artículos & 0.2122 \\
\hline \multirow[t]{2}{*}{ Clase } & $\begin{array}{l}\text { Actividades económicas clasificadas con } Y_{\mathrm{i}}=0 \\
\qquad \text { (38 clases) }\end{array}$ & \\
\hline & 4 clases & $\operatorname{Pr}\left(\hat{Y_{i}}=0\right)>0.5$ \\
\hline 321203 & Fabricación de hilo para coser, bordar y tejer & 0.7080 \\
\hline 323001 & Curtido y acabado de cuero & 0.6543 \\
\hline 321311 & Confecciones de sábanas, manteles, colchas y similares & 0.6402 \\
\hline \multirow[t]{2}{*}{321202} & Hilados de fibras blandas & 0.6144 \\
\hline & 34 clases* & $\operatorname{Pr}\left(\hat{Y}_{i}=0\right)<=0.5$ \\
\hline 321404 & Fabricación de telas de punto & 0.4946 \\
\hline 322012 & Confección de otra ropa interior & 0.2118 \\
\hline 321332 & Tejido de máquina de alfombras y tapetes de fibras blandas & 0.1619 \\
\hline 321208 & $\begin{array}{l}\text { Fabricación de encajes, cintas, etiquetas y otra producción de } \\
\text { pasamanería }\end{array}$ & 0.1564 \\
\hline 324002 & Fabricación de calzado de tela con suela de hule o sintética & 0.1412 \\
\hline 321215 & Fabricación de telas no tejidas & 0.1103 \\
\hline
\end{tabular}

Fuente: Cálculos propios.

* Son las clases más representativas. 
empleos por encima del promedio del subsector, aun cuando están tipificadas en la variable dependiente dicotómica con cero.

Un objetivo adicional de este trabajo es medir cómo se altera la probabilidad condicional de ocurrencia del evento $\left(Y_{i} / X_{i}\right)$ cuando cambia marginalmente la variable explicativa. Para tal efecto, seevaluó la función de densidad para el punto medio, denotada por

$$
\phi\left(\overline{X_{i}} \hat{\beta}\right)=\phi\left(\hat{\beta}_{1}+\hat{\beta}_{2} \overline{X_{i}}\right) \text { equivalente } \phi\left(\bar{X}_{i} \hat{\beta}\right)=\frac{1}{(2 \pi)^{1 / 2}} \lambda^{\frac{\left(-22.1345+1.5730^{*}+12.925\right)^{2}}{2}}
$$

Al efectuar las operaciones correspondientes se obtiene un valor de 0.0867 , que al ser multiplicado por el coeficiente asociado a la demanda efectiva real de 1.5730, arroja un efecto marginal de $X_{i}$ sobrela probabilidad degenerar empleos por arriba del promedio desubsector de $0.1364 \cong 0.14$.

Por otro lado, al analizar la proporción de las predicciones correctas del modelo Probit (Expectation-Prediction), se determina que para 13 clases deactividad económica denotadas por $Y_{i}=1$, el model o estima que hay nueve quetienen una probabilidad mayor a 0.5 de seguir generando empleos por encima del promedio; mientras que las restantes cuatro siguen en condiciones de mantener su potencial pero con probabilidades inferiores a 0.5. De las 38 clases de actividad económica que generan empleos por debajo del promedio del subsector y que son identificadas en la ecuación de regresión por $Y_{i}=0$, el modelo estima que sólo cuatro de ellas tienen una probabilidad superior a 0.5 de generar empleos por encima del promedio.

De las 13 observaciones identificadas con $Y_{i}=1$, las nueve que tienen probabilidad mayor a 0.5 representan el $69.23 \%$ y las cuatro restantes el $30.77 \%$. A su vez, de las 38 clases denotadas por $Y_{i}=0$, las cuatro quetienen una probabilidad mayor a 0.5 representan el $10.53 \%$ y las restantes 34 con probabilidad menor a 0.5 equivalen al $89.47 \%$. Bajo estas consideraciones, el escenario quese vislumbra es quedelas 38 clases de actividad económica que ofrecen puestos de trabajo en menor cuantía queel promedio, 33.83 dlases no aumentarán la ocupación mayor al promedio y 4.17 sí lo harán. Por su parte, de las 13 que si están por arriba del promedio, 8.68 clases lo seguirán logrando y 
4.32 no. En el caso de las 38 observaciones denotadas por $Y_{i}=0$, el $89.02 \%$ son estimaciones correctas, y para $Y_{i}=1$, el $66.80 \%$ son también estimaciones correctas de acuerdo a la evaluación de la capacidad depredicción del modelo.

En el cuadro 3 se aprecia cómo las clases de actividad 321206, 322002, 321322, 324001, 322003, 322006, 322011, 321207 y 321401 son las más importantes en cuanto al efecto que producela demanda efectiva real sobre el nivel de empleo. Dentro de este grupo se observa que las cinco primeras clases más importantes corresponden a la producción de tejido de fibras blandas con 0.9942, confección deropa exterior para caballero hecha en serie con 0.9931 , confección de otros artículos con materiales textiles naturales o sintéticos con 0.9886 , fabricación decalzado principal mente de cuero con 0.9791 y confección de ropa exterior para dama hecha en serie con 0.9535 de probabilidad respectivamente. Otro resultado importante es que delas 38 clases de actividad económica que en 1998 generaron empleos por debajo del promedio, cuatro de ellas presentan probabilidades superiores a 0.5 de ubicarse arriba de la media. Éstas son las clases 321203, 323001, 321311 y 321202, que están asociadas a fabricación de hilo para coser, bordar y tejer con 0.7080 , curtido y acabado de cuero 0.6543 , confecciones de sábanas, manteles, colchas y similares con 0.6402 e hilado de fibras blandas con 0.6144 de probabilidad respectivamente.

\section{Comentariosfinales}

En el presente documento se presenta un análisis de la capacidad de generación de empleos en las diferentes clases de actividad económica que comprenden el subsector 32 de la industria manufacturera en México. Dicha capacidad se determina a partir de la evaluación de la respuesta del mercado laboral a variaciones en la demanda efectiva. La estimación de diferentes modelos probabilísticos produce resultados que indican que el empleo en el subsector 32 está estrechamente relacionado con el comportamiento de la demanda efectiva. Desde estalógica, es importantedestacar quecual quier esfuerzo por diseñar y aplicar una política de empleo debe considerar el papel quedesempeñan los consumidores e inversionistas privados. 
Hoy en día, son los agentes económicos privados los que mayor participación tienen en los mercados de bienes y de servicios a raíz del impulso de los procesos de liberalización comercial y de privatización de las empresas públicas. Así, resulta natural esperar queel sector privado sea el principal generador decrecimiento económico y, consecuentemente, de empleo. Sin embargo, es importante destacar que en la actualidad el sector público, aun acotado, también puede contribuir al crecimiento del empleo a través de una estrategia equilibrada que le permita el evar la demanda efectiva. Tal estrategia, en una perspectiva demediano plazo, pudiera incluir el fortal ecimiento de los ingresos tributarios y no tributarios a partir deal guna reforma fiscal que le permita al Estado aumentar su gasto en la magnitud en que crezcan sus ingresos. Si esto fuera congruente con una adecuada instrumentación dela política monetaria y con un mejoramiento dela productividad total de los factores, muy probablemente se consumaría la estabilización de precios, al mismo tiempo que se generaría un crecimiento económico sano y sostenido.

\section{Bibliografía}

Aláez, Ricardo, 1998, “La política económica en la teoría general de Keynes", en Trimestre Económico, vol. LXV(II), enero-marzo, núm. 257.

Anisi, David, 1988, Trabajar con red, Alianza, Madrid.

Barber J., Williams, 1998, H istoria del pensamiento económico, Alianza Universidad, Madrid.

Banco de M éxico, 2000, Estadísticas sobre índices de precios, México.

Ekelum B., Robert y Herber F. Rober, 1997, H istoria dela teoría económi-

ca y su método, McGraw-Hill, Madrid.

Ehrenberg, R.G. y R. S. Smith, 1999, M odern Labor E conomics: Theory and Public Policy, 7ạ edición, Addison-Wesley, Estados Unidos.

Fremman, C. y L. Soete, 1996, Cambio tecnológico y empleo, Fundación Universidad Empresa, Madrid.

Gujarati, D., 2004, Econometría, 4a edición, McGraw-Hill, México. INEGI, 2000, Censo industrial de la industria manufacturera, México. 
Keynes J., Maynard, 1990, Teoría general de la ocupación, el interés y el dinero, Fondo de Cultura Económica, México.

McConnell, C. y S. Brue, 2002, Contemporary Labour Economics, McGrawHill, Estados Unidos.

Pindyck y Rubinfeld, 1998, M odelos econométricos. M étodos y aplicaciones, 4ạ edición, McGraw-Hill, México.

Artículo recibido el 9 de septiembre de 2004 Artículo aprobado el 11 de marzo de 2005 